\title{
Correlates of psychiatric inpatient admission in a paediatric eating disorder cohort
}

\author{
Matthew Hamilton ${ }^{1}$, Hunna Watson², Sarah Egan ${ }^{4}$, Kimberley Hoiles ${ }^{1 *}$, Emily Harper ${ }^{1}$, Julie McCormack, \\ David Forbes ${ }^{3}$, Chloe Shu ${ }^{1}$ \\ From 2014 ANZAED Conference: Driven Bodies Driven Brains \\ Fremantle, Australia. 22-23 August 2014
}

\section{Objective}

The prevalence and correlates of impending psychiatric inpatient admissions in children and adolescents with eating disorders were examined.

\section{Method}

The sample comprised patients aged 8 to 17 years $(91 \%$ female), with DSM-5 eating disorder diagnosis, categorised as with $(\mathrm{n}=38)$ or without $(\mathrm{n}=247)$ impending psychiatric admission, assessed between 2006 and 2013. The data source was the Helping to Outline Paediatric Eating Disorders (HOPE) Project registry $(\mathrm{N} \sim 1000)$, a prospective, ongoing registry study comprising consecutive paediatric tertiary eating disorder referrals.

\section{Results}

Multivariate analysis of variance and discriminant function analysis were conducted to examine correlates. The prevalence of impending psychiatric admission was $13.3 \%$. Significant group differences were found on psychological, behavioural, and situational correlates. Specifically, suicidal ideation, depressive symptoms, eating pathology, multiple methods of weight control, anxiety, purging behaviours, family functioning, and exercise for shape and weight control.

\section{Conclusions}

Almost 1 in 7 young people with an eating disorder who attended assessment had a presentation needing inpatient psychiatric care, and these individuals could be differentiated from individuals not hospitalised or treated in inpatient medical settings. Implications of these findings include better identification of patients at critical psychiatric risk, earlier recognition and intervention for these patients and more focused assessment of comorbid psychiatric symptoms in specialised eating disorder triage and assessment. Adaptions at the study site to clinical and training protocols will be discussed.

This abstract was presented in the Service Initiatives: Child and Adolescent stream of the 2014 ANZAED Conference.

\section{Authors' details}

${ }^{1}$ Eating Disorders Program, Specialized Child and Adolescent Mental Health Service, Child and Adolescent Health Service, Australia. ${ }^{2}$ UNC Center of Excellence for Eating Disorders, University of North Carolina, Chapel Hill, USA. ${ }^{3}$ School of Paediatrics and Child Health, Faculty of Medicine, Dentistry and Health Sciences, The University of Western Australia, Perth, Australia. ${ }^{4}$ School of Psychology and Speech Pathology, Division of Health Sciences, Curtin University, Perth, Australia.

Published: 24 November 2014

\section{doi:10.1186/2050-2974-2-S1-056}

Cite this article as: Hamilton et al: Correlates of psychiatric inpatient admission in a paediatric eating disorder cohort. Journal of Eating Disorders 2014 2(Suppl 1):056.

\footnotetext{
* Correspondence: Kimberley.Hoiles@health.wa.gov.au

${ }^{1}$ Eating Disorders Program, Specialized Child and Adolescent Mental Health

Service, Child and Adolescent Health Service, Australia

Full list of author information is available at the end of the article
} 